\title{
Design of Decoupled PI Controllers for Two-by-Two Systems *
}

Karl Johan Åström†, Karl Henrik Johansson; and Qing-Guo Wang§

\begin{abstract}
The design of PI controllers for systems with interacting loops is discussed. It is advantageous to deal with the interaction at the loop level, because supervisory control seldom has sufficient bandwidth. A new scheme based on modified scalar PI design and static decoupling is developed, where the frequency characteristic of the coupling between the lower-level loops is taken into account. This leads to a design method emphasizing the trade-off between the individual loop performances and the interaction indices introduced in the paper. The controller is easily implemented, due to its simple configuration based on standard components. A useful observation is that the interaction can be reduced substantially by using set-point weighting. The method is applied to three examples, including a model of a new laboratory system called the quadruple-tank process.
\end{abstract}

\section{Introduction}

Model predictive control (MPC) is becoming the standard technique to solve multi-variable control problems in the process industry $[1,2,3]$. Practically all

*Invited paper to the special issue on PID control of the IEE Proceedings D-Control Theory and Application.

$\dagger$ Department of Automatic Control, Lund University, SE-221 00 Lund, Sweden, kja@control.1th.se.

$\ddagger$ Department of Signals, Sensors and Systems, Royal Institute of Technology, SE-100 44 Stockholm, Sweden, kallej@s3.kth.se. Corresponding author.

$\S$ Department of Electrical Engineering, National University of Singapore, Singapore, elewqg@nus.edu.sg. 
MPC systems are however operating in a supervisory mode with PID controllers at the lower level. A substantial portion of the performance improvement credited to MPC is actually due to improvements in the lower-level PID loops. Interaction among the loops causes difficulties when the lower-level loops are closed. There are some difficulties in dealing with the interaction at the MPC level because the bandwidths of the MPC loops are limited: they operate in supervisory mode with sampling intervals that are longer than in the PID loops. It is therefore of interest to investigate ways of dealing with interaction at the loop level [4]. A preliminary study of this problem is given in the paper. The presentation is restricted to systems with two inputs and two outputs, because such systems are common. Typical examples are boilers, machine direction moisture and basis weight control in paper machines, distillation columns, heat exchangers, and air-conditional systems [5]. The results can be extended to systems with more inputs and outputs.

The approach we take is to investigate standard PID tuning [6] and see what can be achieved by adding simple interactions between the feedback loops. In many cases the performance of the system can be considerably improved, particularly if the coupling in the process is not severe. The proposed scheme is based on a simple static decoupling, which implies that it can be easily implemented at the loop level. The advantage by doing this is that it gives performance enhancement in a frequency range that is normally not dealt with by MPC. Decoupling is a classical tool in multi-variable control. Static and dynamic decoupling are treated in many textbooks in process control, e.g., [7]. Recent contributions to the design of decoupled PID control include the work by Adusumilli et al. [8]. Detuning for multi-variable PID control, as discussed in the paper, was treated in a heuristic setting by Niederlinski [9].

Interaction analysis of multi-variable systems has been an important issue for control structure design (such as input-output pairing) and decentralized control problems. The first quantitative measure of interaction was the relative gain array (RGA) introduced by Bristol [10]. It has been used widely and successfully in the process industries $[11,12]$. The most well known results on the RGA are that a plant with large or negative elements in its RGA is difficult to control and that input and output variables should be paired such that the 
diagonal elements of the RGA are as close as possible to unity [13, 14]. An alternative measure called the steady-state interaction indices was developed by Chang and Davison [15] and it may provide a more accurate analysis of multiloop interaction, especially when the number of inputs and outputs is high. A new interaction index is introduced in this paper.

\section{The Method}

Consider a multi-variable control problem consisting of the design of a linear controller $C$ for a linear stable process $G$. For simplicity assume the process has two inputs and two outputs, so that $G$ is a transfer function of the form

$$
G(s)=\left(\begin{array}{ll}
g_{11}(s) & g_{12}(s) \\
g_{21}(s) & g_{22}(s)
\end{array}\right)
$$

The controller to be designed is a static decoupler combined with a decentralized PID controller with set-point weighting. The control law can be written as

$$
\left(\begin{array}{l}
U_{1}(s) \\
U_{2}(s)
\end{array}\right)=\left(\begin{array}{ll}
d_{11} & d_{12} \\
d_{21} & d_{22}
\end{array}\right)\left(\begin{array}{l}
\bar{c}_{1}(s) Y_{r 1}(s)-c_{1}(s) Y_{1}(s) \\
\bar{c}_{2}(s) Y_{r 2}(s)-c_{2}(s) Y_{2}(s)
\end{array}\right)
$$

where $U$ is the control signal, $Y$ the process output, and $Y_{r}$ the reference. The decoupler

$$
D=\left(\begin{array}{ll}
d_{11} & d_{12} \\
d_{21} & d_{22}
\end{array}\right)
$$

is a constant matrix. The PID controller $\bar{c}_{i}$ is different from $c_{i}$ to allow for set-point weighting [6]. The controllers are of the form

$$
\begin{aligned}
& c_{i}=k_{P i}+\frac{k_{I i}}{s}+k_{D i} s \\
& \bar{c}_{i}=b_{i} k_{P i}+\frac{k_{I i}}{s}
\end{aligned}
$$

where $b_{i}$ is the set-point weight, which in practice often is equal to zero. Here, for simplicity we assume $b_{i}=0$ and PI control, i.e., $k_{D i}=0$. It will be shown that setting $b_{i}=0$ is essential to get good performance in decentralized PID control. 


\subsection{Static Decoupling}

The static decoupler is given by

$$
D=G^{-1}(0)=\frac{1}{\operatorname{det} G(0)}\left(\begin{array}{cc}
g_{22}(0) & -g_{12}(0) \\
-g_{21}(0) & g_{11}(0)
\end{array}\right)
$$

where we have assumed that $G(0)$ is non-singular. The transfer function of the decoupled system is $Q(s)=G(s) D$ where

$$
\begin{aligned}
& q_{11}(s)=\frac{g_{11}(s) g_{22}(0)-g_{12}(s) g_{21}(0)}{\operatorname{det} G(0)} \\
& q_{12}(s)=\frac{g_{12}(s) g_{11}(0)-g_{12}(0) g_{11}(s)}{\operatorname{det} G(0)} \\
& q_{21}(s)=\frac{g_{21}(s) g_{22}(0)-g_{21}(0) g_{22}(s)}{\operatorname{det} G(0)} \\
& q_{22}(s)=\frac{g_{22}(s) g_{11}(0)-g_{21}(s) g_{12}(0)}{\operatorname{det} G(0)}
\end{aligned}
$$

It follows from the construction that $Q(0)$ is the identity matrix. A Taylor series expansion of the transfer function $Q(s)$ for small $|s|$ gives

$$
Q(s) \approx\left(\begin{array}{cc}
1 & \kappa_{12} s \\
\kappa_{21} s & 1
\end{array}\right)
$$

for some constants $\kappa_{12}$ and $\kappa_{21}$. Hence, for low frequencies $\omega$, the diagonal elements are equal to one and the off-diagonal elements are proportional to $i \omega$. If the bandwidth of the decentralized PID controller is sufficiently low, the off-diagonal terms will thus be small and the system will be approximately decoupled.

It is straightforward to see that the closed-loop system can be described by

$$
\left(\begin{array}{cc}
1+q_{11} c_{1} & q_{12} c_{2} \\
q_{21} c_{1} & 1+q_{22} c_{2}
\end{array}\right) Y=\left(\begin{array}{cc}
q_{11} \bar{c}_{1} & q_{12} \bar{c}_{2} \\
q_{21} \bar{c}_{1} & q_{22} \bar{c}_{2}
\end{array}\right) Y_{r}
$$

where we suppressed the dependency on $s$ in the notation. This equation can be written as

$$
Y=\bar{H} Y_{r}
$$


where

$$
\begin{aligned}
& \bar{h}_{11}=\frac{q_{11} \bar{c}_{1}+\left(q_{11} q_{22}-q_{12} q_{21}\right) \bar{c}_{1} c_{2}}{\left(1+q_{11} c_{1}\right)\left(1+q_{22} c_{2}\right)-q_{12} q_{21} c_{1} c_{2}} \\
& \bar{h}_{12}=\frac{q_{12} \bar{c}_{2}}{\left(1+q_{11} c_{1}\right)\left(1+q_{22} c_{2}\right)-q_{12} q_{21} c_{1} c_{2}} \\
& \bar{h}_{21}=\frac{q_{21} \bar{c}_{1}}{\left(1+q_{11} c_{1}\right)\left(1+q_{22} c_{2}\right)-q_{12} q_{21} c_{1} c_{2}} \\
& \bar{h}_{22}=\frac{q_{22} \bar{c}_{2}+\left(q_{11} q_{22}-q_{12} q_{21}\right) c_{1} \bar{c}_{2}}{\left(1+q_{11} c_{1}\right)\left(1+q_{22} c_{2}\right)-q_{12} q_{21} c_{1} c_{2}}
\end{aligned}
$$

The elements in $\bar{H}$ can be simplified by the following argument. The closed-loop bandwidth $\omega_{b}$ is limited by the right half-plane zeros of $G$. If there is a single zero in $z>0$, which is approximately equally distributed between the two loops, then the bandwidth must be less than $0.5 z \mathrm{rad} / \mathrm{s}$, see [16]. It is natural to assume that the multi-variable zeros of $G$ are not within the bandwidth of the closedloop system. (This will also ensure that the decoupler $D$ is well conditioned.) Recall that the zeros of $G$ are given by the solutions to the equation

$$
\operatorname{det} G(s)=g_{11}(s) g_{22}(s)-g_{12}(s) g_{21}(s)=0
$$

If we assume that $|\operatorname{det} G(s)| \gg 0$ for all $|s|<\omega_{b}$, then it follows from $\operatorname{det} Q(s)=$ $\operatorname{det} G(s) \operatorname{det} D$ that $\left|q_{11}(s) q_{22}(s)\right| \gg\left|q_{12}(s) q_{21}(s)\right|$, since $\operatorname{det} Q(s)$ is a meromorphic function and $\operatorname{det} Q(0)=1$. The denominator of the elements $h_{i j}$ can then be approximated by $\left(1+q_{11} c_{1}\right)\left(1+q_{22} c_{2}\right)$. The numerator of $\bar{h}_{11}$ is approximated by $q_{11} \bar{c}_{1}+q_{11} q_{22} \bar{c}_{1} c_{2}=q_{11} \bar{c}_{1}\left(1+q_{22} c_{2}\right)$ and the numerator of $\bar{h}_{22}$ is approximated by $q_{22} \bar{c}_{2}+q_{11} q_{22} c_{1} \bar{c}_{2}=q_{22} \bar{c}_{2}\left(1+q_{11} c_{1}\right)$. The matrix $\bar{H}$ is then approximated by

$$
H=\left(\begin{array}{cc}
\frac{q_{11} \bar{c}_{1}}{1+q_{11} c_{1}} & \frac{q_{12} \bar{c}_{2}}{\left(1+q_{11} c_{1}\right)\left(1+q_{22} c_{2}\right)} \\
\frac{q_{21} \bar{c}_{1}}{\left(1+q_{11} c_{1}\right)\left(1+q_{22} c_{2}\right)} & \frac{q_{22} c_{2}}{1+q_{22} c_{2}}
\end{array}\right)
$$

The structure of the diagonal elements of $H$ is the same as for SISO control design. Therefore, existing techniques [6] can be used for initial suggestions of how to design the decentralized controllers. Sometimes they have to be modified due to the interaction. The off-diagonal elements of $H$ tell us what interaction the SISO designs will lead to. Next, we exploit $H$ to come up with a systematic design procedure for decoupled PI control. 


\section{$2.2 \quad$ Interaction Indices}

Before we discuss the actual design of the PI controllers $c_{i}$ and $\bar{c}_{i}$, let us elaborate further on the off-diagonal elements of $H$ :

$$
\begin{aligned}
h_{12} & =\frac{q_{12} \bar{c}_{2}}{\left(1+q_{11} c_{1}\right)\left(1+q_{22} c_{2}\right)} \\
h_{21} & =\frac{q_{21} \bar{c}_{1}}{\left(1+q_{11} c_{1}\right)\left(1+q_{22} c_{2}\right)}
\end{aligned}
$$

Since the controllers have integral action, we have for small $s$

$$
h_{12}(s) \approx \frac{\kappa_{12} s^{2}}{k_{I 1}}, \quad h_{21}(s) \approx \frac{\kappa_{21} s^{2}}{k_{I 2}}
$$

The interaction is thus very small at low frequencies. To estimate the maximum of the interaction, we observe that

$$
h_{12}=q_{12} \bar{c}_{2} S_{1} S_{2}, \quad h_{21}=q_{21} \bar{c}_{1} S_{1} S_{2}
$$

where $S_{1}=\left(1+q_{11} c_{1}\right)^{-1}$ and $S_{2}=\left(1+q_{22} c_{2}\right)^{-1}$ are the sensitivity for loops when the interaction is neglected. Upper bounds of the interaction terms are thus given by

$$
\begin{gathered}
\left|h_{12}(i \omega)\right| \leq\left|q_{12} \bar{c}_{2}\right| M_{s 1} M_{s 2} \\
\left|h_{21}(i \omega)\right| \leq\left|q_{21} \bar{c}_{2}\right| M_{s 1} M_{s 2}
\end{gathered}
$$

where $M_{s 1}$ and $M_{s 2}$ are the maximum sensitivity for the individual loops. For low frequencies, we have

$$
q_{12}(s) \approx \kappa_{12} s, \quad q_{21}(s) \approx \kappa_{21} s
$$

and

$$
\bar{c}_{1} \approx k_{I 1} / s, \quad \bar{c}_{2} \approx k_{I 2} / s
$$

For low frequencies, we thus find that $q_{12} \bar{c}_{2}$ and $q_{21} \bar{c}_{1}$ are constant. It is therefore natural to introduce the interaction indices

$$
\kappa_{1}=\left|\kappa_{12} k_{I 2}\right| M_{s 1} M_{s 2}, \quad \kappa_{2}=\left|\kappa_{21} k_{I 1}\right| M_{s 1} M_{s 2}
$$

The indices $\kappa_{1}$ and $\kappa_{2}$ describe the interaction of the second loop on the first and vice versa. Note that the indices are products of two terms: one depends on the system and the other is simply the integral gain of the corresponding PI 
controller. Interaction can thus be reduced by reducing the controller gains. We can ensure that the interaction is small by imposing a bound on the controller gains. This is natural because a small $k_{I 2}$ gives less coupling from the responses in the second loop and a large $k_{I 1}$ attenuate disturbances coming from the second loop. Note that $M_{s 1}$ and $M_{s 2}$ also depend on $k_{I 1}$ and $k_{I 2}$, respectively. There is thus a trade-off between $\kappa_{1}$ and $\kappa_{2}$ : if $\left|\kappa_{12}\right|$ and $\left|\kappa_{21}\right|$ are of similar size, then both $\kappa_{1}$ and $\kappa_{2}$ cannot be made small by choosing appropriate integral gains. Either one of them can be made small while keeping the other larger, or both of them have to be kept at a medium level. Which approach to choose, follows from practical considerations of the importance of the individual control loops.

Another convenient measure to be used in the control design is the frequencies at which the interactions $\left|h_{12}(i \omega)\right|$ and $\left|h_{12}(i \omega)\right|$ attain their maxima. These frequencies will determine the bandwidth of the closed-loop system. It is possible to make the following simplifications in order to derive estimates of the frequencies. Estimates of $h_{12}(s)$ and $h_{21}(s)$ for small $s$ are given by (1). For large $s$, the assumption $\left|q_{11}(s) q_{22}(s)\right| \gg\left|q_{12}(s) q_{21}(s)\right|$ used to derive $H$ is still reasonable, because this means that the diagonal elements of $G$ is dominating at high frequencies. Hence, the transfer function $H$ describes the relation between $Y_{r}$ and $Y$ also for large $s$. This leads to that

$$
h_{12}(s) \approx \frac{k_{I 2}}{s^{d_{1}+1}}, \quad h_{21}(s) \approx \frac{k_{I 1}}{s^{d_{2}+1}}
$$

for large $s$, where $d_{1}$ is the pole excess of $q_{12}(s)$ and $d_{2}$ is the pole excess of $q_{21}(s)$. Note that $d_{1}$ and $d_{2}$ are uniquely defined by $G$, and do not depend on the controllers. It follows from (1) and (4) that $\left|h_{12}(i \omega)\right|$ and $\left|h_{21}(i \omega)\right|$ will have maxima. The location of the maximum of $\left|h_{12}(i \omega)\right|$ can, by using (1) and (4), be approximated by the solution of the equation

$$
\frac{\left|\kappa_{12}\right| \omega^{2}}{\left|k_{I 1}\right|}=\frac{\left|k_{I 2}\right|}{\omega^{d_{1}+1}}
$$

The peaks of the anti-diagonal terms $\left|h_{12}(i \omega)\right|$ and $\left|h_{21}(i \omega)\right|$ are thus approximately located at

$$
\omega_{12}=\sqrt[d_{1}+3]{\left|\frac{k_{I 1} k_{I 2}}{\kappa_{12}}\right|}, \quad \omega_{21}=\sqrt[d_{2}+3]{\left|\frac{k_{I 1} k_{I 2}}{\kappa_{21}}\right|}
$$


If the pole excesses of $q_{12}$ and $q_{21}$ are equal to one, then $d_{1}=d_{2}=1$, so that

$$
\omega_{12}=\sqrt[4]{\left|\frac{k_{I 1} k_{I 2}}{\kappa_{12}}\right|}, \quad \omega_{21}=\sqrt[4]{\left|\frac{k_{I 1} k_{I 2}}{\kappa_{21}}\right|}
$$

The interaction indices (3) are derived for PI controllers with set-point weighting $b_{1}=b_{2}=0$. The interactions increase significantly if the controller do not have set-point weighting. If $b_{1}=b_{2}=1$ the interaction measures become

$$
\begin{aligned}
& \bar{\kappa}_{1}=\left|\kappa_{12}\right|\left(\left|k_{P 2}\right| \omega+\left|k_{I 2}\right|\right) M_{s 1} M_{s 2} \\
& \bar{\kappa}_{2}=\left|\kappa_{21}\right|\left(\left|k_{P 1}\right| \omega+\left|k_{I 1}\right|\right) M_{s 1} M_{s 2}
\end{aligned}
$$

Finally, it should be remarked that the estimate (3) can be too conservative if there is a significant difference in the bandwidths of the loops.

\subsection{Design of Decentralized Controllers}

To find the decentralized PID controller, we consider the diagonal terms of the transfer function $Q(s)$. By construction we know that the static gain of all diagonal elements are unity. Standard methods can be used for the design of PI or PID controller for each transfer function $q_{k k}(s)$. The controller parameters that give a PI controller with specified integral gain can be obtained by using dominant pole design, see [17]. Another possibility is to use an optimization technique which maximizes integral gain subject to a robustness constraint. This is discussed for PI controllers in [18] and for PID controllers in [19]. Such a method will give controllers which are optimized with respect to rejection of load disturbances. The design methods will give integral gains $k_{I k}^{0}>0$, which could be used if there were no interactions. The ratios $\lambda_{k}=k_{I k} / k_{I k}^{0}$ are thus measures of performance losses due to the interaction. If $\lambda_{k} \geq 1$ there are no performance losses and the limitations on performance are essentially given by the loop dynamics. If $0 \leq \lambda_{k}<1$ it is necessary to detune the controllers. In this case we need methods to design PID controllers with specifications on the closed-loop bandwidth. There are many methods which can be used for this purpose. Two simple techniques for design of PID controllers are the direct pole-placement design based on reduced-order models and the dominant pole design, see [6] and [17]. 


\subsection{Summary}

The proposed design procedure is summarized in the following algorithm:

1. Find static decoupler $D=G^{-1}(0)$.

2. Select desired interaction indices $\kappa_{1}, \kappa_{2}$ and maximum SISO sensitivities $M_{s 1}, M_{s 2}$.

3. If the specification in Step 2 can be attained by SISO controllers designed through maximization of the integral gains (e.g., [18]), then apply these controllers, else go to Step 4.

4. Either go to Step 2 but make a less demanding design specification, or choose another SISO design method that will fulfill the specification (e.g., dominant pole design [6]).

As illustrated by the algorithm, the primary choice of design for the individual SISO controllers is through maximizing the integral gains. If this result in a closed-loop systems with too large interaction, however, the SISO controllers must be retuned. This can be done either by changing the design specifications (desired $\kappa_{i}$ and $M_{s i}$ ), or by applying another SISO control design method. It is possible to achieve the specifications in Step 2 by choosing SISO designs with sufficiently low bandwidth, which is readily done using dominant pole design. Note that it is easy to combine different SISO design methods and to retune the controllers after simulations, which is illustrated in the examples.

\section{$3 \quad$ Examples}

The control design method is in this section illustrated on three examples from the literature.

\subsection{Rosenbrock's System}

The process

$$
G(s)=\left(\begin{array}{cc}
\frac{1}{s+1} & \frac{2}{s+3} \\
\frac{1}{s+1} & \frac{1}{s+1}
\end{array}\right)
$$




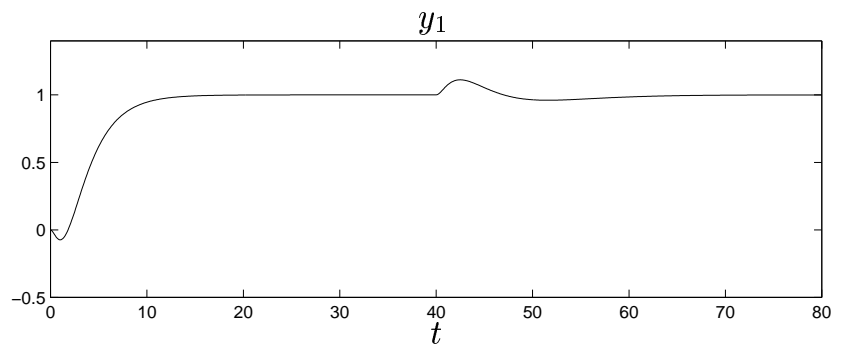

$y_{2}$

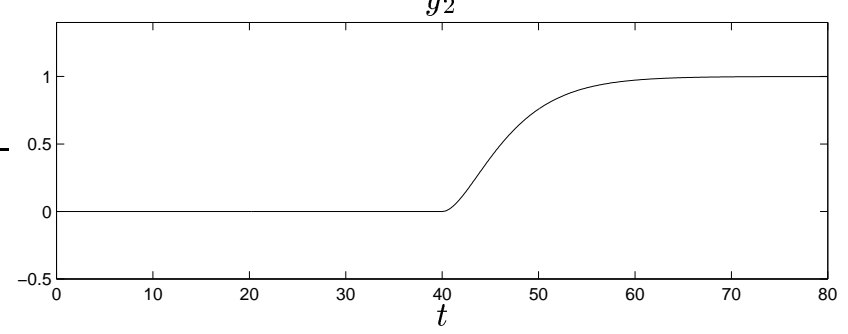

Figure 1: Simulation of the design method applied to Rosenbrock's system. The figure shows the response of the outputs to steps in the command signals. The PI controllers have set-point weighting $b_{1}=b_{2}=0$.

was originally proposed by Rosenbrock [20]. It is an example of a system that looks very easy to control, but which has fundamental limitations because it has a zero $s=1$ in the right half-plane. If we introduce static decoupling, the compensated transfer function becomes

$$
Q(s)=\left(\begin{array}{cc}
\frac{3(1-s)}{(s+1)(s+3)} & \frac{4 s}{(s+1)(s+3)} \\
0 & \frac{1}{s+1}
\end{array}\right)
$$

The interaction is given by $\kappa_{12}=4 / 3$ and $\kappa_{21}=0$. Since $\kappa_{21}=0$, interaction gives no performance limitations for the second loop. There are however limitations because of the right half-plane zero at $s=1$. Designing a PI controller that maximizes integral gain subject to the constraints that the maximum sensitivity $M_{s 1}$ and the maximum complementary sensitivity $M_{p 1}$ are less than $\sqrt{2}$, gives $k_{P 1}=0.245$ and $k_{I 1}=0.248$, see [18]. Since $\kappa_{12}=4 / 3$ there are constraints on the design of the first loop because of the coupling. Requiring that the coupling $\kappa_{1}$ is less than 0.2 and the maximum sensitivity $M_{s 2}$ is less than $\sqrt{2}$, we find that the integral gain of the second loop $k_{I 2}$ must be less than 

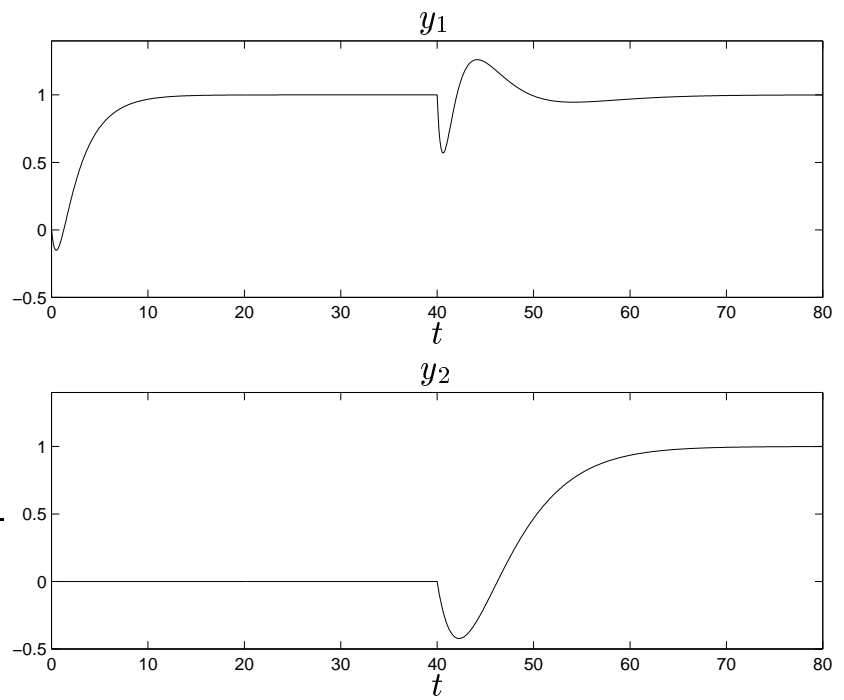

Figure 2: Simulation of controllers without set-point weighting. The simulation is identical to Figure 1 but the controllers have $b_{1}=b_{2}=1$.

$\kappa_{1} /\left(\kappa_{12} M_{s 1} M_{s 2}\right)=0.0750$. To design a PI controller, we use direct pole placement [6] based on the model $q_{22}(s)=(s+1)^{-1}$ and the desired closed-loop characteristic polynomial $s^{2}+2 \zeta \omega_{0} s+\omega_{0}^{2}$. This gives $k_{P 2}=2 \zeta \omega_{0}-1$ and $k_{I 2}=\omega_{0}^{2}$. Requiring that the integral gain is equal to 0.0750 , we find that $\omega_{0}=0.274$. With $\zeta=1$ the controller gains become $k_{P 2}=-0.452$ and $k_{I 2}=0.075$. Figure 1 shows simulations of set-point responses for the closed-loop system. The plots show the proposed design with set-point weighting $\left(b_{1}=b_{2}=0\right)$. A unit step in the set point of the first controller is applied at time $t=0$ and a step in the set point of the second controller is then applied at time $t=40$. Figure 2 shows the step responses for a controller without set-point weighting. The figure clearly indicates the advantage of set-point weighting for multi-variable systems. The reason why there is such a large difference is that the control signal is much smoother with set-point weighting.

The effect of set-point weighting is illustrated also in Figure 3, which shows the frequency response of the closed-loop system with (solid) and without (dashed) set-point weighting. The interaction increases considerably when no set-point weighting is applied. This agrees with the conclusion in previous section; com- 

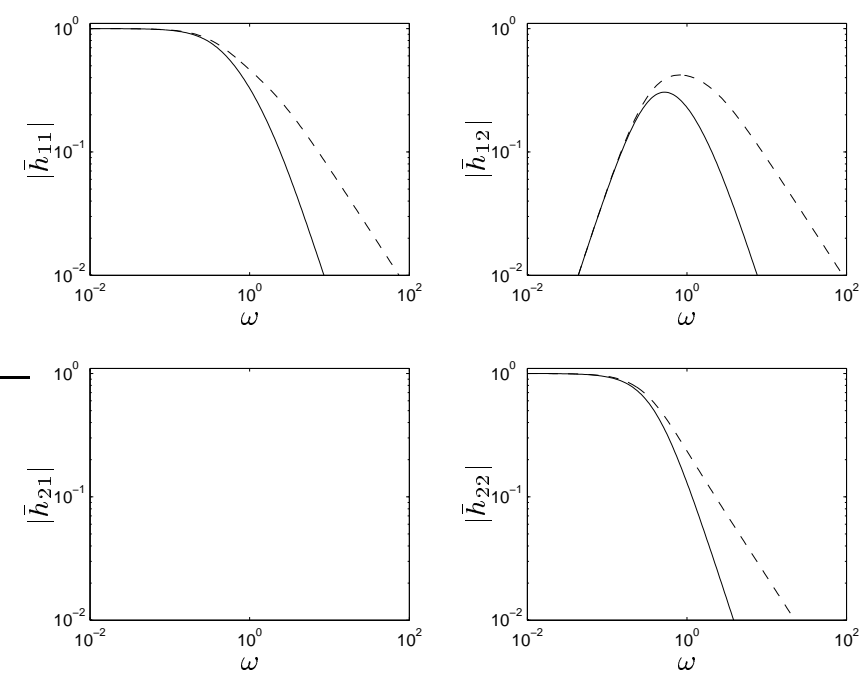

Figure 3: Frequency responses of the closed-loop system with set-point weighting (solid) and without (dashed). Note that without set-point weighting the interaction $\left|\bar{h}_{12}(i \omega)\right|$ is larger and extends to higher frequencies.

pare the interaction indices with and without set-point weighting in Equations (3) and (6).

The maximum of the interaction $\left|\bar{h}_{12}(i \omega)\right|$ in Figure 3 is equal to 0.31 . This should be compared to the interaction index $\kappa_{1}=0.2$. The reason why the estimate $\kappa_{1}$ is lower is that the sensitivities of the system are higher than the design values. The maximum is attained at $\omega=0.52$, while the estimate in (5) predicts 0.41 .

It is interesting to note that the bandwidth of the closed-loop system (as measured by the maximal singular value) is equal to $\omega_{b}=0.49 \mathrm{rad} / \mathrm{s}$, which is close to the limitation imposed by the right half-plane zero at +1 . 


\subsection{Wood-Berry's Binary Distillation Column}

The Wood-Berry binary distillation column plant [21] is a multi-variable system that has been studied extensively. The process has the transfer function

$$
G(s)=\left(\begin{array}{cc}
\frac{12.8 e^{-s}}{16.7 s+1} & \frac{-18.9 e^{-3 s}}{21.0 s+1} \\
\frac{6.60 e^{-7 s}}{10.9 s+1} & \frac{-19.4 e^{-3 s}}{14.4 s+1}
\end{array}\right)
$$

The transfer function of the statically compensated system is

$$
Q(s)=\left(\begin{array}{cc}
\frac{12.8 e^{-s}}{16.7 s+1} & \frac{-18.9 e^{-3 s}}{21.0 s+1} \\
\frac{6.60 e^{-7 s}}{10.9 s+1} & \frac{-19.4 e^{-3 s}}{14.4 s+1}
\end{array}\right)\left(\begin{array}{cc}
0.1570 & -0.1529 \\
0.0534 & -0.1036
\end{array}\right)
$$

A series expansion for small $s$ gives

$$
Q(s) \approx\left(\begin{array}{cc}
1-11.7 s & -12.31 s \\
-0.5138 s & 1-17.3 s
\end{array}\right)
$$

The interaction is thus given by $\kappa_{12}=-12.31$ and $\kappa_{21}=-0.5138$. The time constants of the reduced-order models are $T_{1}=11.7$ and $T_{2}=17.3$. Let the desired maximum sensitivity for the individual loops be equal to $M_{s 1}=M_{s 2}=$ $\sqrt{2}$. Requiring that the interaction indices $\kappa_{1}$ and $\kappa_{2}$ should both be less than 0.20 , the integral gains should be $k_{I 1}<0.19$ and $k_{I 2}<0.0081$. This gives the corresponding crossover frequencies

$$
\begin{aligned}
& \omega_{01}<\sqrt{k_{I 1} / T_{1}}=\sqrt{0.19 / 11.7}=0.12 \\
& \omega_{02}<\sqrt{k_{I 2} / T_{2}}=\sqrt{0.0081 / 17.3}=0.022
\end{aligned}
$$

We have $\omega_{01} L=0.36$ and $\omega_{02} L=0.066$. The bandwidths are thus well below the limitations $\omega L<0.7$ imposed by the the largest time delay of the system $(L=3)$, see [16]. Note that in this case there is a significant difference between the bandwidths of the loops.

With these frequencies and the time delays present in the system, it is quite reasonable to approximate the time delays with first order lags. The model reduction should thus work reasonably well in this case. Choosing $\zeta_{1}=\zeta_{2}=$ $\zeta=0.707$ we find that the proportional gains are

$$
\begin{aligned}
& k_{P 1}=2 \zeta \omega_{01}-1=1.1338 \\
& k_{P 2}=2 \zeta \omega_{02}-1=-0.4699
\end{aligned}
$$



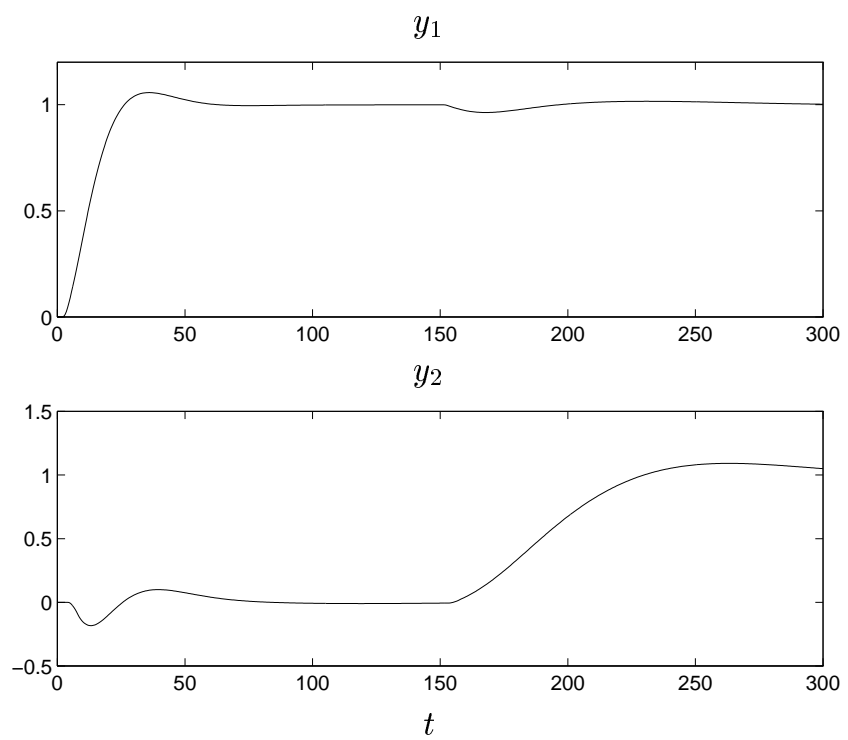

Figure 4: Simulation of the design method applied to the Wood-Berry distillation column. The figure shows the response of the outputs to steps in the command signals.

In Figure 4 we show the step responses from set point to process output for the system. Analyzing the figure we find that the response in $y_{1}$ caused by a step in the set point $y_{r 2}$ is quite small. It thus seems possible to increase the bandwidth in the second loop. After some experimentation we found that a reasonable value is $\omega_{02}=0.1$, which gives that the controller parameters for the second loop become $k_{P 2}=0.7$ and $k_{I 2}=0.123$. The time responses are shown in Figure 5. A comparison with Figure 4 shows that the response speed of the second loop has been increased considerably without increasing the interaction too much. This also shows that the method lends itself very well to tuning. The step responses in Figure 5 compare favorably with designs obtained by other methods.

\subsection{Quadruple-Tank Process}

Consider the quadruple-tank process in Figure 6. This lab process has been used to illustrate many issues in multi-variable control [22]. The target is to control 

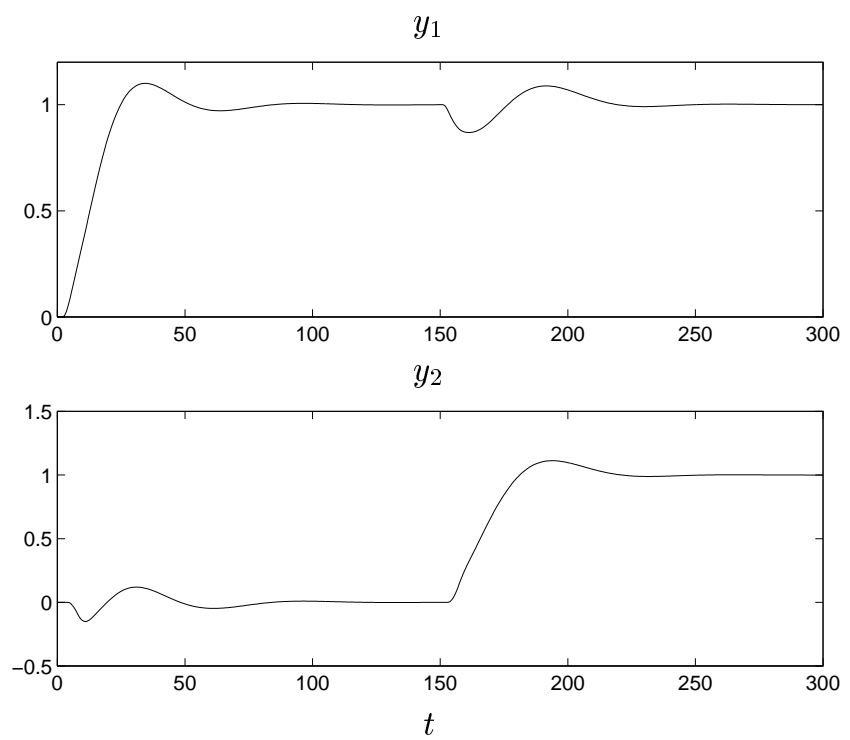

Figure 5: Simulation of the design method applied to the Wood-Berry distillation column. The bandwidth of the second loop has been increased to $\omega_{02}=0.1$.

the level in the lower two tanks with two pumps. The process inputs are $u_{1}$ and $u_{2}$ (input voltages to the pumps) and the outputs are $y_{1}$ and $y_{2}$ (voltages from level measurement devices). The linearized dynamics for the process (which we limit the discussion to here) is given by

$$
G(s)=\left(\begin{array}{cc}
\frac{\gamma_{1} \alpha_{1}}{1+s T_{1}} & \frac{\left(1-\gamma_{2}\right) \alpha_{1}}{\left(1+s T_{3}\right)\left(1+s T_{1}\right)} \\
\frac{\left(1-\gamma_{1}\right) \alpha_{2}}{\left(1+s T_{4}\right)\left(1+s T_{2}\right)} & \frac{\gamma_{2} \alpha_{2}}{1+s T_{2}}
\end{array}\right)
$$

where

$$
T_{i}=\frac{A_{i}}{a_{i}} \sqrt{\frac{2 q_{i}^{0}}{g}} \quad i=1, \ldots, 4
$$

and $\alpha_{1}=T_{1} k_{1} k_{c} / A_{1}$ and $\alpha_{2}=T_{2} k_{2} k_{c} / A_{2}$. Here $A_{i}$ is the cross-section of Tank $i, a_{i}$ the cross-section of the outlet hole, $q_{i}^{0}$ is the steady-state water level, $k_{i}$ is the gain of Pump $i, k_{c}$ the measurement gain, and $g$ acceleration of gravity. The parameters $\gamma_{1}, \gamma_{2} \in(0,1)$ are determined from how the valves are set prior to an experiment: the flow to Tank 1 is proportional to $\gamma_{1}$ and the flow to Tank 4 is proportional to $\left(1-\gamma_{1}\right)$, and similarly for $\gamma_{2}$ with respect to Tank 2 


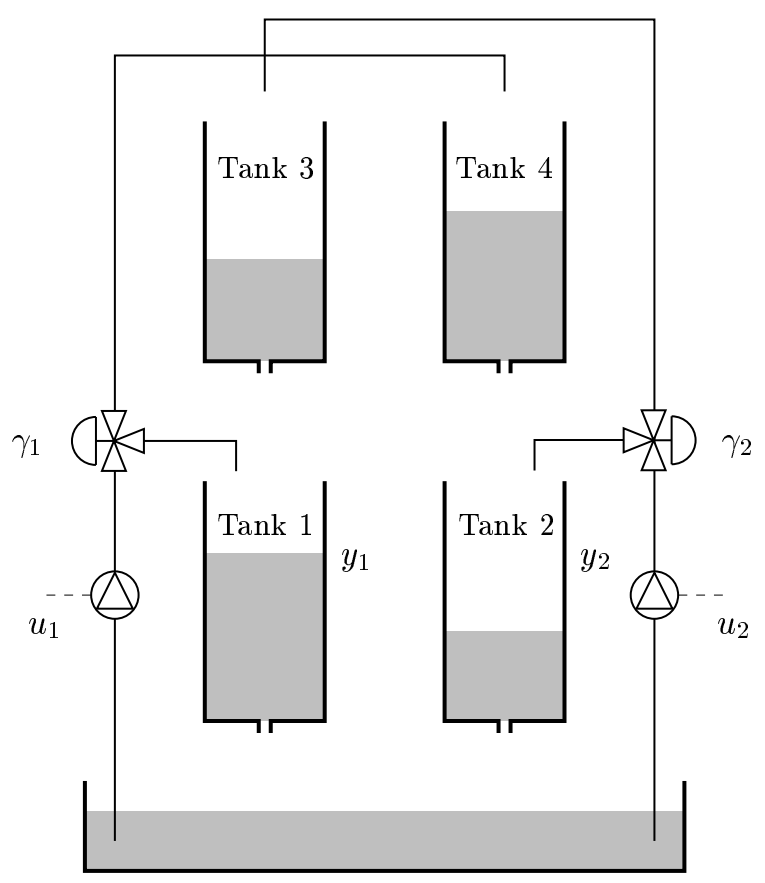

Figure 6: Schematic diagram of the quadruple-tank process. The water levels in Tank 1 and Tank 2 are controlled by two pumps. The flow ratios $\gamma_{1}$ and $\gamma_{2}$ set by the valves determine the location of the transmission zero for the linearized model. The zero can be put in either the left or the right half-plane.

and Tank 3. Since

$$
\begin{aligned}
\operatorname{det} G(s) & =\frac{\alpha_{1} \alpha_{2}}{\gamma_{1} \gamma_{2} \prod_{i=1}^{4}\left(1+s T_{i}\right)} \\
& \times\left[\left(1+s T_{3}\right)\left(1+s T_{4}\right)-\frac{\left(1-\gamma_{1}\right)\left(1-\gamma_{2}\right)}{\gamma_{1} \gamma_{2}}\right]
\end{aligned}
$$

the transfer matrix $G$ has two finite zeros for $\gamma_{1}, \gamma_{2} \in(0,1)$. The system is non-minimum phase for

$$
0<\gamma_{1}+\gamma_{2}<1
$$

and minimum phase for

$$
1<\gamma_{1}+\gamma_{2}<2
$$

Hence, by changing a single valve we can make the multi-variable level control problem more or less difficult. The quadruple-tank process is thus ideal for 
evaluating control designs. This is done next for the proposed decoupled PID control design method. We restrict the discussion to the case

$$
T_{1}=\cdots=T_{4}=T>0, \quad \alpha_{1}=\alpha_{2}=1
$$

The decoupled system is then given by

$$
\begin{aligned}
Q(s) & =G(s) D=G(s) G^{-1}(0) \\
& =\frac{1}{(1+s T)^{2}}\left(\begin{array}{cc}
1-s T \beta & s T \frac{\gamma_{1}\left(\gamma_{2}-1\right)}{\gamma_{1}+\gamma_{2}-1} \\
s T \frac{\gamma_{2}\left(\gamma_{1}-1\right)}{\gamma_{1}+\gamma_{2}-1} & 1-s T \beta
\end{array}\right)
\end{aligned}
$$

where $\beta=\gamma_{1} \gamma_{2} /\left(1-\gamma_{1}-\gamma_{2}\right)$. Note that

$$
q_{11}(s)=q_{22}(s)=\frac{1-s T \beta}{(1+s T)^{2}}
$$

has a right half-plane zero whenever $G$ has a (multi-variable) right half-plane zero. As the zero approaches the origin (i.e., as $\beta$ tends to $+\infty$ ), the achievable closed-loop bandwidth vanishes. This is also reflected by the interaction indices

$$
\kappa_{12}=\frac{T \gamma_{1}\left(1-\gamma_{2}\right)}{1-\gamma_{1}-\gamma_{2}}, \quad \kappa_{21}=\frac{T \gamma_{2}\left(1-\gamma_{1}\right)}{1-\gamma_{1}-\gamma_{2}}
$$

which tend to $+\infty$ as the zero approaches the origin. Note that $\kappa_{12}$ and $\kappa_{21}$ are positive if the system $G(s)$ is minimum phase and negative if it is not.

Suppose the valves of the quadruple-tank system are set such that $\gamma_{1}=\gamma_{2}=$ $\gamma \in(0,1 / 2)$. The system is then non-minimum phase with the right half-plane zero located in $(1-2 \gamma) /(T \gamma)$. Let us study the particular case when $T=1$ and $\gamma=1 / 3$. Requiring that the coupling $\kappa=\kappa_{1}=\kappa_{2}$ is less than 0.2 and the maximum sensitivity $M_{s 1}=M_{s 2}$ is equal to $\sqrt{2}$ lead through Equation (3) to the design constraint $k_{I}=k_{I 1}=k_{I 2}<0.15$. (Note that we due to the symmetry of the process have chosen identical SISO controllers $c_{1}=c_{2}$.)

A pole placement design for

$$
q_{11}(s)=q_{22}(s) \approx \frac{1-s T \beta}{1+s 2 T}
$$

gives

$$
\begin{aligned}
k_{P} & =\frac{2 \omega_{0}^{2} T^{2} \beta+4 \zeta \omega_{0} T-1}{\omega_{0}^{2} T^{2} \beta^{2}+2 \zeta \omega_{0} T \beta+1} \\
k_{I} & =\frac{\omega_{0}^{2} T(2+\beta)}{\omega_{0}^{2} T^{2} \beta^{2}+2 \zeta \omega_{0} T \beta+1}
\end{aligned}
$$


where

$$
s^{2}+2 \zeta \omega_{0} s+\omega_{0}^{2}
$$

denotes the (approximate) denominator polynomial of $h_{11}(s)=h_{22}(s)$.

The right half-plane zero in $(1-2 \gamma) /(T \gamma)=1$ suggests that $\omega_{0}$ should be considerably smaller than one. Let us compare designs with $\omega_{0}=0.1,0.2, \ldots, 0.6$. The controller parameters are given as follows:

\begin{tabular}{l|llllll}
$\omega_{0}$ & 0.1 & 0.2 & 0.3 & 0.4 & 0.5 & 0.6 \\
\hline$k_{P}$ & -0.678 & -0.371 & -0.079 & 0.197 & 0.460 & 0.708 \\
$k_{I}$ & 0.022 & 0.085 & 0.150 & 0.150 & 0.150 & 0.150
\end{tabular}

Recall the design constraint $k_{I}<0.15$. When $\omega_{0} \geq 0.3$, the integral gains obtained through the pole placement design violates the constraint. The applied integral gain is then fixed to 0.150 as shown in the table.

The estimates of $\kappa=\kappa_{1}=\kappa_{2}$ and $\bar{\omega}=\omega_{12}=\omega_{21}$ using Equations (3) and (5), respectively, are given in the following table. The corresponding true values

$$
\begin{aligned}
& \bar{h}_{\max }=\max _{\omega}\left|h_{12}(i \omega)\right|=\max _{\omega}\left|h_{21}(i \omega)\right| \\
& \omega_{\max }=\arg \max _{\omega}\left|h_{12}(i \omega)\right|=\arg \max _{\omega}\left|h_{21}(i \omega)\right|
\end{aligned}
$$

are also presented.

\begin{tabular}{l|llllll}
$\omega_{0}$ & 0.1 & 0.2 & 0.3 & 0.4 & 0.5 & 0.6 \\
\hline$\kappa$ & 0.030 & 0.113 & 0.200 & 0.200 & 0.200 & 0.200 \\
$\bar{\omega}$ & 0.165 & 0.323 & 0.429 & 0.429 & 0.429 & 0.429 \\
\hline $\bar{h}_{\max }$ & 0.158 & 0.190 & 0.179 & 0.104 & 0.076 & 0.071 \\
$\omega_{\max }$ & 0.100 & 0.211 & 0.320 & 0.404 & 0.706 & 0.977
\end{tabular}

The interaction index $\kappa$ is a reasonable upper estimate of the true interaction $\bar{h}_{\max }$ for $\omega_{0}=0.2,0.3,0.4$. The estimate $\bar{\omega}$ of the frequency at which the maximum is attained is quite close for all $\omega_{0}=0.1, \ldots, 0.6$. Figure 7 shows the frequency responses for the elements in $\bar{H}$.

The achievable bandwidth with the proposed decoupled PID design is surprisingly close to the limitation imposed by the right half-plane zero at +1 . This is illustrated in Figure 8, which shows the frequency responses for the maximum singular valued of the closed-loop system for $\omega_{0}=0.1,0.2, \ldots, 0.6$. Figure 9 shows the corresponding responses to steps in the command signals. 

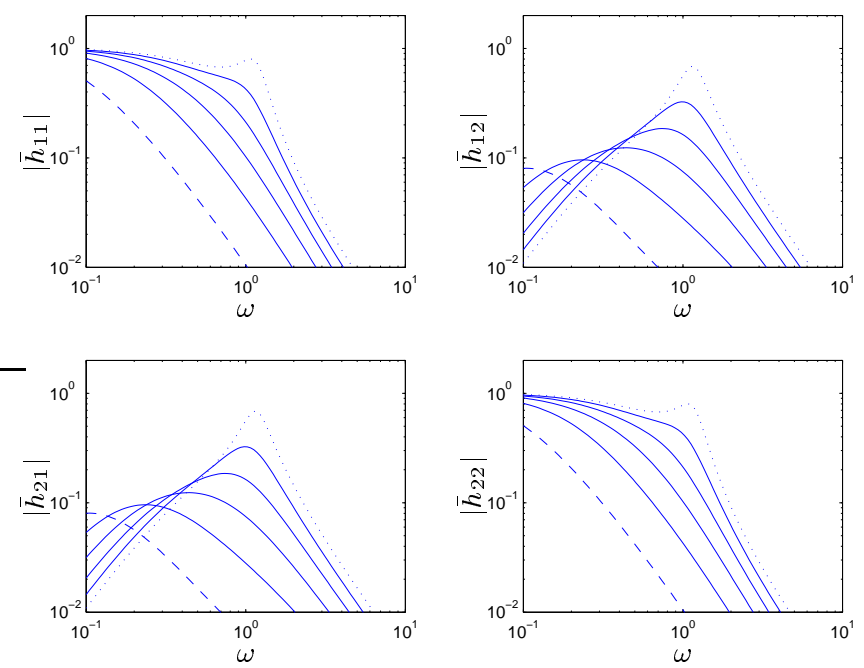

Figure 7: Frequency responses of the closed-loop system for designs with $\omega_{0}=$ $0.1,0.2, \ldots, 0.6$. The dashed line corresponds to $\omega_{0}=0.1$ and the dotted line to $\omega_{0}=0.6$.

Note that both Rosenbrock's system and the quadruple-tank system have a right half-plane zero in +1 . In both cases decoupled PID control succeeds giving a closed-loop system with a bandwidth of approximately 0.5 which is close to the limit derived in [16]. The result for the Wood-Berry's binary distillation column is similar. The examples thus suggest that the simple rule of thumb for SISO systems may be extended to multi-variable systems. In some cases it is important to also take the direction of the multi-variable right half-plane zero into account [23]. For example, zero location and direction can be quantified in terms of a trade-off between set-point response and interaction [24].

\section{Conclusions}

The suggested control scheme is a simple multi-variable controller consisting of a static decoupler and two single-loop PI controllers. In spite of its simplicity the controller can deal with many practical control problems. It is also easy to implement in virtually any DCS system. A method for finding the parameters of 


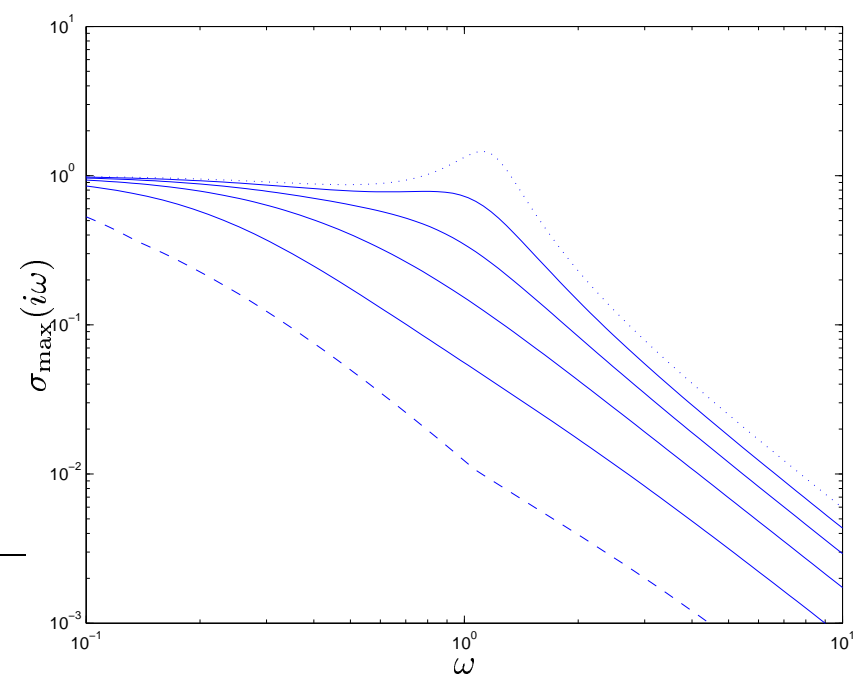

Figure 8: Maximum singular value of the closed-loop system illustrating the bandwidth of the system for designs with $\omega_{0}=0.1,0.2, \ldots, 0.6$.

the controller has been developed in the paper. The key assumption is that the interaction is not too severe. This is captured quantitatively by the interaction indices $\kappa_{1}$ and $\kappa_{2}$. Possible detuning of the PID controllers was quantified in terms of these indices. An important observation is that the interaction is reduced substantially by using set-point weighting $b_{1}=b_{2}=0$.

The interaction indices $\kappa_{1}$ and $\kappa_{2}$ depend on both the process and the controller. Note that the RGA reflects the interaction under very tight control. The indices introduced in this paper capture the fact that interaction can be reduced by detuning the loops. Furthermore, note that our interaction indices also take dynamics into account. For the decoupled system $Q(s)=G(s) D$ the RGA is equal to the identity matrix, while the dynamic RGA [12] for small $|s|$ has approximately the diagonal elements $1-\kappa_{12} \kappa_{21} s^{2}$ and the anti-diagonal elements $\kappa_{12} \kappa_{21} s^{2}$ (with $\kappa_{i j}$ as defined in Section 2). 

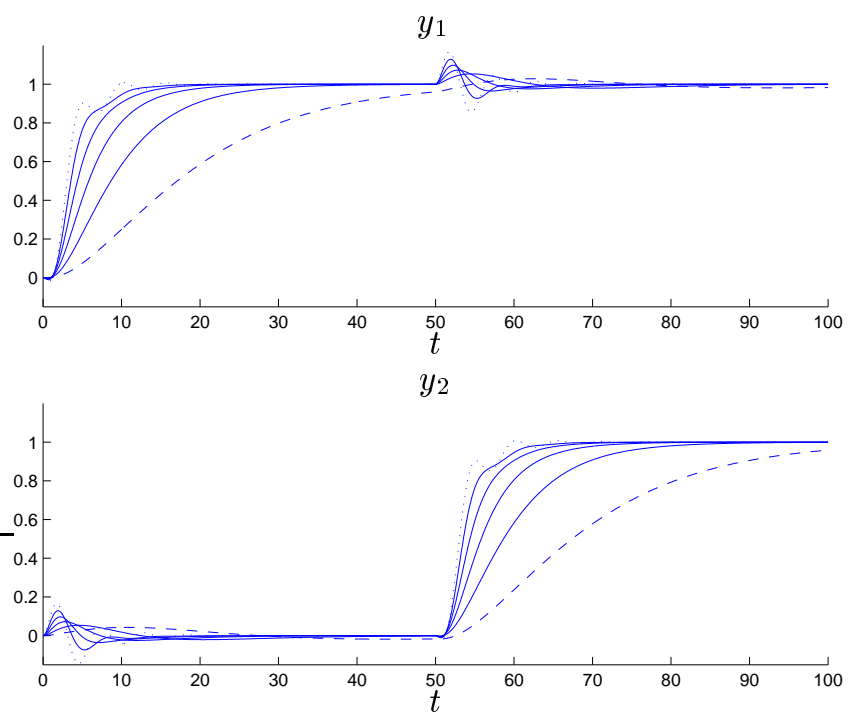

Figure 9: Step response experiments for the linearized quadruple-tank process controlled using decoupled PID control. The plots correspond to $\omega_{0}=$ $0.1,0.2, \ldots, 0.6$.

\section{References}

[1] S. J. Qin and T. A. Badgwell. An overview of industrial model predictive control technology. In J. C. Kantor, editor, Conference on Chemical Process Control CPC V, pages 232-256, London, 1996. CACHE, Elsevier.

[2] E. F. Camacho and C. Bordons. Model Predictive Control. Springer, London, 1999.

[3] J. M. Maciejowski. Predictive Control with Constraints. Pearson Education, 2000.

[4] Y. Lee, S. Park, and J. H. Lee. On interfacing model predictive controllers with low-level loops. In Preprints. 14th World Congress of IFAC, volume N, pages 313-318, Beijing, China, 1999.

[5] W. L. Luyben. Process modeling, simulation, and control for chemical engineers. McGraw-Hill, New York, 1990. 
[6] K. J. Åström and T. Hägglund. PID Controllers: Theory, Design, and Tuning. Instrument Society of America, Research Triangle Park, NC, 1995.

[7] D. E. Seborg, T. F. Edgar, and D. A. Mellichamp. Process Dynamics and Control. Wiley, New York, 1989.

[8] S. Adusumilli, D. E. Rivera, S. Dash, and K. Tsakalis. Integrated MIMO identification and robust PID controller design through loop shaping. In Proc. of ACC, Philadelphia, PA, 1998.

[9] A. Niederlinski. A heuristic approach to the design of linear multivariable interacting control systems. Automatica, 7:691-701, 1971.

[10] E. Bristol. On a new measure of interaction for multivariable process control. IEEE Trans. Autom. Control, 11:133, 1966.

[11] F. G. Shinsky. Controlling Multivariable Processes. Instrument Society of America, Research Triangle Park, NC, 1981.

[12] T. J. McAvoy. Interaction Analysis: Principles and Applications. Instrument Society of America, Research Triangle Park, NC, 1983.

[13] P. Grosdidier, M. Morari, and B. R. Holt. Closed-loop properties from steady-state gain information. Industrial and Engineering Chemistry Fundamentals, 24:221-235, 1985.

[14] S. Skogestad and M. Morari. Implications of large RGA elements on control performance. Industrial and Engineering Chemistry Research, 26:2323$2330,1985$.

[15] T. N. Chang and E. J. Davison. Steady-state interaction indices for decentralized unknown systems. In Proc. IEEE Conference on Decision and Control, volume 2, pages 881-887, 1986.

[16] K. J. Åström. Limitations on control system performance. European Journal on Control, 6:2-20, 2000.

[17] P. Persson and K. J. Åström. Dominant pole design - a unified view of PID controller tuning. In Preprints 4th IFAC Symposium on Adaptive Systems in Control and Signal Processing, pages 127-132, Grenoble, France 1992. 
[18] K. J. Åström, H. Panagopoulos, and T. Hägglund. Design of PI controllers based on non-convex optimization. Automatica, 34(5):585-601, 1998.

[19] H. Panagopoulos. PID-Control. Design, Extension, Application. PhD thesis, Dept. of Automatic Control, Lund University, Sweden, 2000.

[20] H. H. Rosenbrock. State-Space and Multivariable Theory. Nelson, London, U.K., 1970.

[21] R. K. Wood and M. W. Berry. Terminal composition control of a binary distillation column. Chem. Engng. Sci., 28:1707-1717, 1973.

[22] K. H. Johansson. The Quadruple-Tank Process-a multivariable laboratory process with an adjustable zero. IEEE Transactions on Control Systems Technology, 8(3):456-465, May 2000.

[23] M. M. Seron, J. H. Braslavsky, and G. C. Goodwin. Fundamental Limitations in Filtering and Control. Springer-Verlag, 1997.

[24] K. H. Johansson. Interaction bounds in multivariable control systems. In 14 th International Federation of Automatic Control World Congress, Beijing, China, 1999. 